\title{
Determinants of prenatal care use and HIV testing during pregnancy: a population- based, cross-sectional study of 7080 women of reproductive age in Mozambique
}

Sanni Yaya ${ }^{{ }^{*+}}$ D, Olanrewaju Oladimeji ${ }^{2,3,4}$, Kelechi Elizabeth Oladimeji ${ }^{5}$ and Ghose Bishwajit ${ }^{6{ }^{*+}}$

\begin{abstract}
Background: In low-income countries with poor coverage of healthcare services such as Mozambique, antenatal care serves as a vital tool for providing life-saving and cost-effective services for pregnant mothers. Nonetheless, many countries in Africa, including Mozambique, are struggling to attain an optimum level of antenatal care (at least 4 visits) utilisation among pregnant women. In the present study, we aimed to assess the sociodemographic and economic factors associated with antenatal care use in Mozambique.

Methods: Cross-sectional data from the latest round of Mozambique Demographic and Health Survey (2011) on women aged $15-49$ years $(n=7080)$ were analysed. The outcome measures were early and adequate antenatal visit and HIV tests during the last pregnancy. Data were analysed using descriptive and multivariate regression methods. The predictor variables included various demographic (e.g. age, parity), empowerment (e.g. type of employment, household wealth status) and sociocultural factors (e.g. ethnicity, religion).

Results: Of the 7080 women whose data was analyzed, 15.3 and $60.1 \%$ had early and adequate ANC visits respectively while $75.4 \%$ received HIV test during ANC visits. The odds of early ANC visits were higher [OR $=1.300,95 \% \mathrm{Cl}=1.062$, 1.592] among women in the rural areas compared with those in the urban areas. However, participants in rural areas had lower odds $[O R=0.788,0.687,0.902]$ of receiving HIV tests during ANC visits. Women in the urban areas with secondary $[\mathrm{OR}=1.296,95 \% \mathrm{Cl}=1.007,1.666]$ and higher $[\mathrm{OR}=1.663,95 \% \mathrm{Cl}=1.052,2.628]$ education had higher odds of having early ANC visit. Those in the higher wealth quintiles also had significantly increased odds of using all three types of ANC indicators, particularly for rural women in the highest wealth quintile $[\mathrm{OR}=4.776,95 \% \mathrm{Cl}=1.250,18.24]$. Being within the higher wealth quintiles was found to significantly increase the odds of using all three types of ANC indicators, particularly women from rural areas with highest wealth quintile $[\mathrm{OR}=4.776,95 \% \mathrm{Cl}=1.250,18.24]$.

Conclusion: About two-fifth of the women in Mozambique are not using adequate antenatal care and about and a quarter do not take HIV tests during pregnancy. The sources of low and unequal use of these vital health services might be rooted in women's socioeconomic status and cultural issues that require special policy and research attention.
\end{abstract}

Keywords: Maternal health, Antenatal care, HIV test, Global health, Mozambique

\footnotetext{
*Correspondence: sanni.yaya@georgeinstitute.ox.ac.uk;

brammaputram@yahoo.fr

${ }^{\dagger}$ Sanni Yaya and Ghose Bishwajit are joint first authors.

${ }^{1}$ The George Institute for Global Health, 75 George Street, Oxford OX1 2BQ,

United Kingdom

${ }^{6}$ School of International Development and Global Studies, University of

Ottawa, Ottawa, Ontario K1N 6N5, Canada

Full list of author information is available at the end of the article
}

(c) The Author(s). 2019 Open Access This article is distributed under the terms of the Creative Commons Attribution 4.0 International License (http://creativecommons.org/licenses/by/4.0/), which permits unrestricted use, distribution, and reproduction in any medium, provided you give appropriate credit to the original author(s) and the source, provide a link to the Creative Commons license, and indicate if changes were made. The Creative Commons Public Domain Dedication waiver (http://creativecommons.org/publicdomain/zero/1.0/) applies to the data made available in this article, unless otherwise stated. 


\section{Background}

As a part of overall human development process, countries in sub-Saharan Africa have been striving to reduce the high maternal and child mortality rates. Driven by the Millennium Development Goals (MDGs), many countries in the region have been able to make concrete improvements in this regard $[1,2]$. Nonetheless, the sub-Saharan African countries continue to account for a staggering $66 \%$ of all pregnancy and childbirth related deaths in the world [3]. Maternal mortality is a complex indicator that can be triggered by a multitude of factors. However, it is widely acknowledged that the bulk of the health issues arising from pregnancy can actually be circumvented by taking precautionary measures provided through antenatal care techniques $[4,5]$. Antenatal care (ANC) provides a unique window of opportunity to ensure a healthy pregnancy through ensuring healthy weight gain and proper nutrition (e.g. intake of iron and folic acid), taking necessary immunizations packages such as tetanus toxoid and antimalarial drugs, controlling gestational diabetes, hypertension, and many other pregnancy related medical conditions $[4,6,7]$.

With the rising prevalence of childhood HIV, the impact of making ANC visits has become more prominent than ever as ANC service now includes HIV counselling and testing. Women who fail to attend ANC are less likely to be aware of the risks associated with mother-tochild transmission of HIV through pregnancy, childbirth, and breastfeeding. In the low-income settings, ANC visits allow women to check their HIV status which is an effective way to prevent mother-to-child transmission of HIV [8-10], the key contributor to childhood HIV in Africa $[11,12]$. Exposure to ANC services not only increases the scope of taking the preventive measures and diagnosis of high-risk pregnancy and other pregnancy related conditions, but also the likelihood of choosing health facility delivery. Studies have demonstrated that majority of the maternal and child deaths occur during unassisted deliveries and in the neonatal period [13-15], which can be avoided if women are given the necessary consultations through the ANC package. This is particularly important for countries like Mozambique where a large proportion of the population live below the poverty line.

Nonetheless, a large proportion of the women across the developing world remain deprived of the ANC services [9]. The inequality in accessing and utilizing healthcare services is further attributed to various behavioral, cultural, economic, and sociodemographic factors at individual level and remoteness of health facility, inadequate infrastructure and skilled human resource for healthcare at community level [16-20]. Understanding a country's specific predictors of healthcare utilisation is essential for making targeted approaches to combat pregnancy related deaths among women and newborns. However, there is no such study or report available for Mozambique, and thus the factors behind the low utilisation of ANC services are not known. Therefore, the present study was conducted to explore the factors associated with ANC service utilisation using nationallyrepresentative data from Mozambique Demographic and Health Survey (DHS) conducted in 2011. This was a cross-sectional survey that provided information on a wide range of demographic and socioeconomic variables.

\section{Methodology}

\section{Data source}

Data for this study were collected from the sixth round of Mozambique Demographic and Health Survey. The survey was conducted in 2011 by the country's national statistical institute (Instituto Nacional de Estatística) and ministry of health (MISAU). Techincal support and assistance was provided by United States Agency for International Development of the United America (USAID) with ICF International. Sample population included eligible men (15-54 years) and women (15-49 years) residing in households in urban and rural areas excluding institutions such as hospitals, hotels, dorms. Data collection was done through direct interviews using Tablet-type computer (Computer-Assisted Personal Interview) system and this process lasted from June 2011 to November 2011. Sampling was done using multistate cluster technique which involves stratifying the provinces into primary sampling units (PSUs), and then selection of households from the households form each PSUs. Of the 13,964 households initially selected, a total of 13,718 women were finally interviewed, resulting in a $99 \%$ response rate. These details are available from the final report of Mozambique 2011 DHS: Ministerio da Saude (MISAU), Instituto Nacional de Estatística (INE) e ICF International (ICFI). Moçambique Inquérito Demográfico e de Saúde 2011. Calverton, Maryland, USA: MISAU, INE e ICFI.

\section{Variables used in the analysis \\ Outcome variables.}

The outcome variables of interest were: 1) timing of first antenatal care, 2) frequency of antenatal care, 3) HIV testing during ANC visit. All of these items were assessed based on the self-report of the participant for the latest childbirth occurring within the last five years from the survey. Timing of first antenatal care was classified as early (if within the first trimester) and late (if beyond the first trimester) [3]. Frequency of antenatal care visits was defined as adequate (at least four visits) and inadequate (less than four visits) as per World Health Organization recommendation. HIV testing during ANC visit was classified as Yes (had HIV tests) and No (had no HIV tests). 


\section{Explanatory variables}

Selection of explanatory variables was guided by Andersen's behavioral model of health service utilisation which postulates that healthcare utilisation is a function of three major factors: 1) predisposing factors, 2) enabling factors and 3) need factors [21]. For this study, the data were secondary and hence the selection of the explanatory variables in line with the behavioral model was not possible. Based on the availability of variables in the dataset, the following was included in the analysis: Age (15-19, 20-24, 25-29, 30-34, 35-39, 40-44, 45-49); Residency (Urban, Rural); Education (No Education, Primary, Secondary, Higher); Husbands education (No Education, Incomplete Primary, Incomplete Secondary, Higher); Employment (Not Working, Professional/Technical/Managerial, Agricultural - Self Employed); Wealth quintile (Poorest, Poorer, Middle, Richer, Richest); Access to electronic media including TV and radio (No, Yes); Heard of FP on internet (No, Yes); Religion (Islam, Other); Ethnicity (Emakhuwa, Portugais, Xichangana, Cisena, Elomwe, Cindau, Xitswa, Other); Parity $(1-5,>5)$; Household head (Male, Female); Child wanted (Wanted Then, Wanted No More).

\section{Data management and analysis}

The dataset was cleaned by applying the inclusion criteria: experience of at least 1 childbirth in the preceding 5 years and analysed with Stata version 14.. As the surveys used cluster sampling techniques, all analyses were adjusted for this by using the svy command [22]. This command uses the information on sampling weight, strata, and primary sampling unit provided with the datasets. Sample characteristics were described as percentages. Prevalence of timing and adequacy of antenatal care was presented as bar charts. Following that, binary logistic regression models were used to estimate odds ratio (with 95\%CIs) of using these services. Results of three outcome variables were presented in separate tables, each divided into three subsamples: overall, urban and rural. Model fit statistics were run after the regression analyses using the variance inflation factor (VIF) command. No multi-collinearity was detected as VIF values were below 10 for all the models. All tests were two-tailed and were considered significant at alpha value of $5 \%$.

\section{Ethical considerations}

The analyses were done using publicly available data from demographic health surveys. Ethical procedures were the responsibility of the institutions that commissioned, funded, or managed the surveys. All DHS surveys are approved by ICF international as well as an Institutional Review Board (IRB) in respective country to ensure that the protocols are in compliance with the U.S. Department of Health and Human Services regulations for the protection of human subjects. an informed oral consent is obtained from each respondent and a parent or guardian provides consent for an adolescent under 18 years. The description of DHS consent process is available from https://www.dhsprogram.com/WhatWe-Do/Protecting-the-Privacy-of-DHS-Survey-Respondents.cfm.

\section{Results}

\section{Summarized characteristics of the study participants}

Table 1 shows that a greater proportion of the participants were aged $25-29$ years (25.01\%), rural residents (63.16\%), had primary level education (51.5\%), had no employment (53.59\%), from households with richest wealth quintile (25.92\%), had access to electronic media (71.69\%), didn't hear about family planning on internet (98.88\%), followers of Islam (71.12\%), of Xichangana ethnicity (31.87\%), had 1-5 children (81.78\%), from male-headed households (64.90\%), and wanted the last child (79.86\%).

Table 1 also shaows that only $15.3 \%$ of the women made early contact, $60.1 \%$ had adequate number of ANC visits and $75.4 \%$ had HIV test during ANC visits.

Presented in Tables 2 and 3, are the sociodemographic predictors of early and adequate ANC visits respectively. As opposed to urban areas, in the rural areas women within aged 20-24 years significantly had lower odds of having early $[\mathrm{OR}=0.634,95 \% \mathrm{CI}=0.467,0.862]$ ANC visits (Table 2). The odds of early ANC visits were higher [OR = $1.300,95 \% \mathrm{CI}=1.062,1.592]$ in the rural areas compared with urban (Table 2). Women in the urban areas with secondary $[\mathrm{OR}=1.296,95 \% \mathrm{CI}=1.007,1.666]$ and higher $[\mathrm{OR}=1.663,95 \% \mathrm{CI}=1.052,2.628]$ education had higher odds of having early ANC visit. Similar associations with educational status were observed for adequate ANC visit and taking HIV tests as well. No significant association was observed for adequate use of ANC (Table 3) among women aged 20-24 years, whereas a positive association was found between the age groups of 20-24 years and taking HIV test (Table 4).

Husband's educational status also showed a positive association with women's adequate ANC visits (Table 3) and taking HIV tests (Table 4). Women who had employment in agriculture were more likely to make early ANC in the both urban $[\mathrm{OR}=1.513,95 \% \mathrm{CI}=1.019$, $2.247]$ and rural $[\mathrm{OR}=1.232,1.039,1.461]$ areas.

For HIV tests, however, a significant negative association was found in urban women. Women in the higher wealth quintiles also have significantly increased odds of using all three types ANC indicators, particularly for rural women in the highest wealth quintile $[\mathrm{OR}=4.776$, $95 \% \mathrm{CI}=1.250,18.24]$. Having access to electronic media showed an inverse relationship with adequate ANC use $[\mathrm{OR}=0.744,95 \% \mathrm{CI}=0.660,0.840]$, but positive relationship with taking $\mathrm{HIV}$ tests $[\mathrm{OR}=1.233,95 \% \mathrm{CI}=1.102$, 1.381]. Significant religion and ethnic variations were 
Table 1 Summarized characteristics of the women aged 15-49 years $(N=7080)$

\begin{tabular}{|c|c|c|c|c|c|}
\hline & \\
\hline Variable & Freq. & Percent & Variable & Freq. & Percent \\
\hline Age & & & Cindau & 426 & 6.02 \\
\hline $15-19$ & 813 & 11.48 & Xitswa & 368 & 5.20 \\
\hline $20-24$ & 1771 & 25.01 & Other & 2104 & 29.72 \\
\hline $25-29$ & 1697 & 23.97 & Parity & & \\
\hline $30-34$ & 1319 & 18.63 & $1-5$ & 5790 & 81.78 \\
\hline $35-39$ & 935 & 13.21 & $>5$ & 1290 & 18.22 \\
\hline $40-44$ & 394 & 5.56 & Sex of household head & & \\
\hline $45-49$ & 151 & 2.13 & Male & 4595 & 64.90 \\
\hline Residency & & & Female & 2485 & 35.00 \\
\hline Urban & 2608 & 36.84 & Last child wanted & & \\
\hline Rural & 4472 & 63.16 & Wanted Then & 5654 & 79.86 \\
\hline Education & & & Wanted No More & 1426 & 20.14 \\
\hline No Education & 2139 & 30.21 & Timing of ANC visit & & \\
\hline Primary & 3646 & 51.50 & Early & 5997 & 84.7 \\
\hline Secondary & 1,22 & 17.23 & Late & 1083 & 15.3 \\
\hline Higher & 75 & 1.06 & Frequency of ANC visit & & \\
\hline Husband's education & & & $<4$ & 2825 & 39.9 \\
\hline No Education & 1731 & 26.36 & 4 or more & 4255 & 60.1 \\
\hline Primary & 3272 & 49.82 & HIV testing during ANC & & \\
\hline Secondary & 1422 & 21.65 & Yes & 1742 & 24.6 \\
\hline Higher & 143 & 2.18 & No & 5338 & 75.4 \\
\hline
\end{tabular}

Occupation

Not Working 3794

Professional/Technical/Managerial $\quad 1269$

Agricultural - Self Employed 2017

Wealth index

Poorest

1067

Poorer

Middle

Richer

Richest

Electronic Media access

No

Yes

Religion

Islam

Other

Ethnicity

Emakhuwa

1241

Portugais

Xichangana

Cisena

Elomwe

\section{7}

1392

676

266
Table 1 Summarized characteristics of the women aged 15-49 years $(N=7080)$ (Continued)

found for taking HIV testing services. Parity and household head's sex didn't show any noteworthy associations with the outcome variables.

A significantly negative association was found between child wanted-ness and the use of the ANC services. For early ANC visit, the negative association was true for women in the rural areas only $[\mathrm{OR}=0.615,95 \% \mathrm{CI}=$ $0.488,0.775]$, and for taking HIV test the result was significant only in the urban $[\mathrm{OR}=0.599,95 \% \mathrm{CI}=0.428$, 0.839] areas. Whereas, having unwanted child showed a negative association with adequate ANC visits both in urban $[\mathrm{OR}=0.770,95 \% \mathrm{CI}=0.626,0.948]$ and rural $[\mathrm{OR}=$ $0.662,95 \% \mathrm{CI}=0.544,0.806]$ areas. Women in rural areas had lower odds $[\mathrm{OR}=0.788,0.687,0.902]$ of receiving HIV tests during ANC visits (Table 4). As opposed to making adequate ANC vsits, having access to electronic media showed a positive relationship with taking HIV tests $[\mathrm{OR}=1.233,95 \% \mathrm{CI}=1.102,1.381]$.

\section{Discussion}

Pregnancy represents a crucial phase of women's reproductive life that requires a set of special care and services that can be accessed through antenatal care. This is especially true for women in low-income settings such as Mozambique where poor insurance coverage 
Table 2 Predictors of making early ANC visits among women aged 15-49 years in Mozambique $(n=7080)$

\begin{tabular}{|c|c|c|c|}
\hline & Overall & Urban & Rural \\
\hline Age (15-19) & 1 & 1 & 1 \\
\hline $20-24$ & $0.711^{*}[0.547,0.924]$ & $0.970[0.572,1.645]$ & $0.634^{* *}[0.467,0.862]$ \\
\hline $25-29$ & $0.976[0.755,1.261]$ & $1.112[0.662,1.867]$ & $0.955[0.707,1.291]$ \\
\hline $30-34$ & $1.005[0.769,1.313]$ & $1.356[0.801,2.293]$ & $0.878[0.638,1.207]$ \\
\hline $35-39$ & $1.071[0.796,1.441]$ & $1.370[0.782,2.397]$ & $0.961[0.669,1.379]$ \\
\hline $40-44$ & $1.081[0.766,1.526]$ & $1.644[0.852,3.170]$ & $0.879[0.581,1.330]$ \\
\hline $45-49$ & $1.037[0.658,1.634]$ & $1.060[0.424,2.646]$ & $0.994[0.583,1.696]$ \\
\hline Residency (Urban) & 1 & NA & NA \\
\hline Rural & $1.300^{*}[1.062,1.592]$ & & \\
\hline Education (No Education) & 1 & 1 & 1 \\
\hline Primary & $1.181[0.993,1.405]$ & $0.929[0.683,1.265]$ & $1.332^{* *}[1.077,1.648]$ \\
\hline Secondary & $1.256^{*}[1.054,1.497]$ & $1.296^{*}[1.007,1.666]$ & $1.158[0.900,1.491]$ \\
\hline Higher & $1.873^{* *}[1.256,2.793]$ & $1.663^{*}[1.052,2.628]$ & $2.233 \mathrm{~N}[0.858,5.808]$ \\
\hline Husband's education (No Education) & 1 & 1 & 1 \\
\hline Incomplete Primary & $1.214[0.940,1.569]$ & $1.291[0.869,1.917]$ & $1.193[0.847,1.680]$ \\
\hline Incomplete Secondary & $1.070[0.907,1.261]$ & $1.114[0.874,1.419]$ & $1.044[0.829,1.316]$ \\
\hline Higher & $1.210[0.924,1.583]$ & $1.438^{*}[1.002,2.064]$ & $0.977[0.638,1.498]$ \\
\hline Employment (Not Working) & 1 & 1 & 1 \\
\hline Professional//Technical/Managerial & $1.036[0.884,1.214]$ & $1.115[0.903,1.378]$ & $0.930[0.725,1.194]$ \\
\hline Agricultural - Self Employed & $1.325^{* * *}[1.141,1.538]$ & $1.513^{*}[1.019,2.247]$ & $1.232^{*}[1.039,1.461]$ \\
\hline Wealth quintile (Poorest) & 1 & 1 & 1 \\
\hline Poorer & $1.052[0.891,1.243]$ & $0.710[0.377,1.337]$ & $1.096[0.921,1.306]$ \\
\hline Middle & $1.014[0.848,1.213]$ & $0.914[0.550,1.518]$ & $1.032[0.848,1.256]$ \\
\hline Richer & $1.048[0.829,1.325]$ & $0.887[0.556,1.415]$ & $0.932[0.652,1.332]$ \\
\hline Richest & $1.493^{* *}[1.143,1.951]$ & $1.141[0.710,1.835]$ & $4.776^{*}[1.250,18.24]$ \\
\hline Media access (No) & 1 & 1 & 1 \\
\hline Yes & $0.920[0.765,1.107]$ & $1.157[0.692,1.934]$ & $0.880[0.719,1.077]$ \\
\hline Religion (Islam) & 1 & 1 & 1 \\
\hline Other & $1.166[0.719,1.888]$ & $1.486[0.771,2.865]$ & $0.757[0.357,1.603]$ \\
\hline Ethnicity (Emakhuwa) & 1 & 1 & 1 \\
\hline Portugais & $0.661^{* * * *}[0.542,0.807]$ & $0.857[0.614,1.195]$ & $0.562^{* * * *}[0.436,0.724]$ \\
\hline Xichangana & $1.183[0.927,1.509]$ & $1.126[0.796,1.594]$ & $1.259[0.886,1.790]$ \\
\hline Cisena & $1.114[0.955,1.298]$ & $1.057[0.787,1.420]$ & $1.087[0.903,1.308]$ \\
\hline Elomwe & $1.105[0.874,1.397]$ & $1.266[0.787,2.035]$ & $1.002[0.762,1.319]$ \\
\hline Cindau & $1.168[0.951,1.435]$ & $1.205[0.909,1.597]$ & $1.185[0.861,1.632]$ \\
\hline Xitswa & $0.965[0.814,1.145]$ & $1.051[0.704,1.567]$ & $0.951[0.785,1.151]$ \\
\hline Other & $0.944[0.726,1.228]$ & $1.073[0.527,2.185]$ & $0.906[0.679,1.208]$ \\
\hline Parity (1-5) & 1 & 1 & 1 \\
\hline$>5$ & $0.827^{*}[0.691,0.989]$ & $0.733[0.526,1.020]$ & $0.901[0.723,1.123]$ \\
\hline Household head's sex (Male) & 1 & 1 & 1 \\
\hline Female & $0.984[0.835,1.160]$ & $1.090[0.876,1.357]$ & $0.867[0.669,1.122]$ \\
\hline Child wanted (Wanted Then) & 1 & 1 & 1 \\
\hline Wanted No More & $0.705^{* * * *}[0.591,0.841]$ & $0.847[0.641,1.120]$ & $0.615^{* * * *}[0.488,0.775]$ \\
\hline Pseudo $R^{2}$ & 0.022 & 0.029 & 0.026 \\
\hline
\end{tabular}

Exponentiated coefficients; $95 \%$ confidence intervals in brackets.

${ }^{*} p<0.05,{ }^{* *} p<0.01,{ }^{* * *} p<0.001$ 
Table 3 Predictors of making adequate ANC visits among women aged 15-49 years in Mozambique $(n=7080)$

\begin{tabular}{|c|c|c|c|}
\hline & Overall & Urban & Rural \\
\hline Age (15-19) & 1 & 1 & 1 \\
\hline $20-24$ & $0.953[0.786,1.155]$ & $1.031[0.742,1.433]$ & $0.916[0.722,1.162]$ \\
\hline $25-29$ & $1.068[0.878,1.299]$ & $1.171[0.836,1.640]$ & $1.013[0.796,1.291]$ \\
\hline $30-34$ & $1.001[0.812,1.234]$ & $1.233[0.853,1.784]$ & $0.895[0.692,1.157]$ \\
\hline $35-39$ & $1.118[0.885,1.413]$ & $1.500[0.993,2.267]$ & $0.971[0.729,1.295]$ \\
\hline $40-44$ & $1.098[0.818,1.472]$ & $1.106[0.652,1.876]$ & $1.065[0.747,1.519]$ \\
\hline $45-49$ & $1.354[0.908,2.021]$ & $2.157[0.878,5.297]$ & $1.178[0.746,1.859]$ \\
\hline Residency (Urban) & 1 & & \\
\hline Rural & $1.021[0.884,1.181]$ & & \\
\hline Education (No Education) & 1 & 1 & 1 \\
\hline Primary & $1.149^{*}[1.012,1.304]$ & $1.282[0.956,1.720]$ & $1.083[0.939,1.248]$ \\
\hline Secondary & $1.847^{* * *}[1.476,2.313]$ & $1.871^{* * *}[1.301,2.691]$ & $2.161^{* * * *}[1.509,3.093]$ \\
\hline Higher & $2.790^{*}[1.141,6.827]$ & $2.828^{*}[1.105,7.241]$ & $1.205[0.918,1.487]$ \\
\hline Husband's education (No Education) & 1 & 1 & 1 \\
\hline Incomplete Primary & $1.107[0.974,1.257]$ & $1.002[0.762,1.317]$ & $1.142[0.988,1.321]$ \\
\hline Incomplete Secondary & $1.219^{*}[1.016,1.462]$ & $1.186[0.876,1.605]$ & $1.183[0.924,1.514]$ \\
\hline Higher & $1.970^{*}[1.111,3.493]$ & $2.012^{*}[1.072,3.775]$ & $1.317[0.137,12.63]$ \\
\hline Employment (Not Working) & 1 & 1 & 1 \\
\hline Professional/Technical/Managerial & $1.159[0.989,1.357]$ & $1.136[0.918,1.406]$ & $1.253[0.981,1.601]$ \\
\hline Agricultural - Self Employed & $1.039[0.918,1.176]$ & $1.023[0.767,1.364]$ & $1.052[0.914,1.211]$ \\
\hline Wealth quintile (Poorest) & 1 & 1 & 1 \\
\hline Poorer & $1.163[0.980,1.379]$ & $1.573[0.890,2.783]$ & $1.132[0.946,1.355]$ \\
\hline Middle & $1.404^{* * * *}[1.182,1.669]$ & $1.623[1.000,2.635]$ & $1.332^{* *}[1.105,1.606]$ \\
\hline Richer & $1.566^{* * *}[1.302,1.885]$ & $1.408[0.894,2.215]$ & $1.496^{* * *}[1.206,1.854]$ \\
\hline Richest & $2.100^{* * * *}[1.641,2.686]$ & $1.955^{* *}[1.207,3.166]$ & $2.871^{* * * *}[1.916,4.302]$ \\
\hline Media access (No) & 1 & 1 & 1 \\
\hline Yes & $0.744^{* * *}[0.660,0.840]$ & $0.662^{* * *}[0.504,0.870]$ & $0.787^{* * * *}[0.687,0.903]$ \\
\hline Religion (Islam) & 1 & 1 & 1 \\
\hline Other & $0.933[0.829,1.050]$ & $1.105[0.888,1.377]$ & $0.868[0.753,1.000]$ \\
\hline Ethnicity (Emakhuwa) & 1 & 1 & 1 \\
\hline Portugais & $1.760^{* * * *}[1.326,2.335]$ & $1.781^{* *}[1.219,2.601]$ & $2.478^{* *}[1.327,4.626]$ \\
\hline Xichangana & $1.504^{* * * *}[1.226,1.845]$ & $1.448^{*}[1.033,2.029]$ & $1.716^{* * *}[1.308,2.251]$ \\
\hline Cisena & $1.376^{* *}[1.126,1.680]$ & $1.875^{* *}[1.274,2.759]$ & $1.215[0.959,1.541]$ \\
\hline Elomwe & $1.486^{* *}[1.123,1.967]$ & $1.706[0.870,3.342]$ & $1.419^{*}[1.039,1.939]$ \\
\hline Cindau & $2.285^{* * *}[1.773,2.946]$ & $2.140^{*}[1.135,4.032]$ & $2.313^{* * *}[1.746,3.064]$ \\
\hline Xitswa & $1.326^{*}[1.014,1.732]$ & $1.376[0.774,2.447]$ & $1.312[0.967,1.780]$ \\
\hline Other & $1.580^{* * *}[1.352,1.846]$ & $1.760^{* * *}[1.290,2.401]$ & $1.544^{* * *}[1.286,1.855]$ \\
\hline Parity (1-5) & 1 & 1 & 1 \\
\hline$>5$ & $1.014[0.860,1.195]$ & $0.864[0.623,1.198]$ & $1.087[0.896,1.319]$ \\
\hline Household head's sex (Male) & 1 & 1 & 1 \\
\hline Female & $1.053[0.942,1.179]$ & $0.900[0.741,1.092]$ & $1.117[0.972,1.283]$ \\
\hline Child wanted (Wanted Then) & 1 & 1 & 1 \\
\hline Wanted No More & $0.710^{* * *}[0.617,0.818]$ & $0.770^{*}[0.626,0.948]$ & $0.662^{* * * *}[0.544,0.806]$ \\
\hline Pseudo $R^{2}$ & 0.046 & 0.047 & 0.041 \\
\hline
\end{tabular}

Exponentiated coefficients; $95 \%$ confidence intervals in brackets.

${ }^{*} p<0.05,{ }^{* *} p<0.01,{ }^{* * *} p<0.001$ 
Table 4 Predictors of HIV testing during ANC visits among women aged 15-49 years in Mozambique $(n=7080)$

\begin{tabular}{|c|c|c|c|}
\hline & Overall & Urban & Rural \\
\hline Age (15-19) & 1 & 1 & 1 \\
\hline $20-24$ & $1.232^{*}[1.034,1.468]$ & $1.129[0.687,1.855]$ & $1.385^{*}[1.056,1.816]$ \\
\hline $25-29$ & $1.422^{* * *}[1.191,1.697]$ & $1.531[0.910,2.578]$ & $1.688^{* * * *}[1.275,2.231]$ \\
\hline $30-34$ & $1.376^{* * *}[1.139,1.662]$ & $1.806^{*}[1.000,3.261]$ & $1.521^{* *}[1.130,2.047]$ \\
\hline $35-39$ & $1.283^{*}[1.035,1.589]$ & $1.232[0.651,2.331]$ & $1.626^{* *}[1.163,2.273]$ \\
\hline $40-44$ & $1.217[0.932,1.588]$ & $1.016[0.444,2.325]$ & $1.371[0.901,2.087]$ \\
\hline $45-49$ & $1.149[0.799,1.652]$ & $0.651[0.192,2.212]$ & $1.302[0.755,2.248]$ \\
\hline Residency (Urban) & 1 & & \\
\hline Rural & $0.788^{* * *}[0.687,0.902]$ & & \\
\hline Education (No Education) & 1 & 1 & 1 \\
\hline Primary & $1.373^{* * *}[1.225,1.538]$ & $2.011^{* * *}[1.371,2.950]$ & $1.338^{* * *}[1.132,1.581]$ \\
\hline Secondary & $1.394^{* * *}[1.176,1.652]$ & $3.086^{* * *}[1.733,5.493]$ & $4.574^{* * * *}[2.604,8.035]$ \\
\hline Higher & $1.144[0.697,1.879]$ & $6.053[0.641,57.18]$ & $1.531[0.810,2.416]$ \\
\hline Husband's education (No Education) & 1 & 1 & 1 \\
\hline Incomplete Primary & $1.225^{* * *}[1.088,1.378]$ & $1.401[0.956,2.051]$ & $1.306^{* *}[1.103,1.548]$ \\
\hline Incomplete Secondary & $1.453^{* * * *}[1.252,1.687]$ & $2.549^{* * * *}[1.577,4.120]$ & $1.350[0.992,1.836]$ \\
\hline Higher & $1.373^{*}[1.006,1.874]$ & $1.214[0.355,4.150]$ & $1.126[0.854,1.832]$ \\
\hline Employment (Not Working) & 1 & 1 & 1 \\
\hline Professional//Technical/Managerial & $0.861^{*}[0.748,0.991]$ & $1.784^{* *}[1.167,2.726]$ & $0.702^{*}[0.519,0.950]$ \\
\hline Agricultural - Self Employed & $0.744^{* * * *}[0.668,0.829]$ & $0.857[0.580,1.265]$ & $0.574^{* * *}[0.487,0.675]$ \\
\hline Wealth quintile (Poorest) & 1 & 1 & 1 \\
\hline Poorer & $1.062[0.933,1.209]$ & $1.691[0.883,3.241]$ & $0.999[0.817,1.222]$ \\
\hline Middle & $1.241^{* *}[1.082,1.422]$ & $1.779^{*}[1.011,3.131]$ & $1.217[0.983,1.506]$ \\
\hline Richer & $1.429^{* * * *}[1.216,1.679]$ & $1.955^{*}[1.141,3.348]$ & $1.533^{* *}[1.180,1.992]$ \\
\hline Richest & $1.959^{* * *}[1.576,2.436]$ & $2.534^{* *}[1.380,4.651]$ & $3.376^{* * *}[1.834,6.218]$ \\
\hline Media access (No) & 1 & 1 & 1 \\
\hline Yes & $1.233^{* * *}[1.102,1.381]$ & $1.107[0.761,1.609]$ & $1.111[0.947,1.303]$ \\
\hline Religion (Islam) & 1 & 1 & 1 \\
\hline Other & $0.868^{*}[0.767,0.983]$ & $0.946[0.677,1.321]$ & $0.821^{*}[0.697,0.967]$ \\
\hline Ethnicity (Emakhuwa) & 1 & 1 & 1 \\
\hline Portugais & $2.098^{* * *}[1.737,2.533]$ & $3.349^{* *}[1.602,7.000]$ & $1.994[0.910,4.367]$ \\
\hline Xichangana & $2.072^{* * *}[1.714,2.505]$ & $3.006^{* * *}[1.763,5.125]$ & $1.689^{* *}[1.222,2.334]$ \\
\hline Cisena & $1.381^{* * *}[1.206,1.581]$ & $1.902^{*}[1.104,3.275]$ & $2.655^{* * *}[2.030,3.473]$ \\
\hline Elomwe & $0.418^{* * * *}[0.315,0.555]$ & $0.243^{* * *}[0.111,0.533]$ & $1.326^{* * *}[1.153,1.526]$ \\
\hline Cindau & $1.415^{* *}[1.099,1.821]$ & $1.281[0.847,1.938]$ & $2.249^{* * *}[1.831,2.762]$ \\
\hline Xitswa & $6.637^{* * *}[4.489,9.813]$ & $6.122^{*}[1.393,26.90]$ & $8.257^{* * * *}[5.379,12.67]$ \\
\hline Other & $3.168^{* * *}[2.263,4.434]$ & $3.143^{*}[1.012,9.755]$ & $3.778^{* * *}[2.565,5.565]$ \\
\hline Parity (1-5) & 1 & 1 & 1 \\
\hline$>5$ & $1.064[0.922,1.229]$ & $1.013[0.622,1.650]$ & $0.963[0.767,1.210]$ \\
\hline Household head's sex (Male) & 1 & 1 & 1 \\
\hline Female & $1.067[0.952,1.196]$ & $0.856[0.621,1.179]$ & $1.286^{* *}[1.085,1.524]$ \\
\hline Child wanted (Wanted Then) & 1 & 1 & 1 \\
\hline Wanted No More & $0.643^{*}[0.447,0.925]$ & $0.599^{* *}[0.428,0.839]$ & $1.144[0.894,1.464]$ \\
\hline Pseudo $R^{2}$ & 0.190 & 0.197 & 0.121 \\
\hline
\end{tabular}

Exponentiated coefficients; $95 \%$ confidence intervals in brackets.

${ }^{*} p<0.05,{ }^{* *} p<0.01,{ }^{* * *} p<0.001$ 
and high out-of-pocket expenditure can prevent the optimum use of these services. Research evidence is therefore necessary to understand the sociodemographic inequalities in the utilisation of ANC services in order to inform the ongoing programs working to meet the maternal health related targets such as Sustainable Development Goals. The current study provides an insight on the prevalence and predictors of the three crucial components of maternal healthcare including early and adequate antenatal care visits and use of HIV testing services among pregnant women in Mozambique. The findings indicate that only $15.3 \%$ of women made their first ANC visit within the first trimester, three-fifth had adequate ANC visits and three-quarter took HIV test during pregnancy. The prevalence of early ANC visits is lower than that of Ghana where $88 \%$ of the sample women received ANC care in 2014 [23]. In a multicountry study based on demographic and Health Survey data, Ghada et al. reported that the prevalence of having adequate ANC visits were Cameroon (62.7\%), Senegal (51.2\%), Uganda (48.5) [24]. Data on timing of the ANC visits is less commonly reported. In Ethiopia, a nationally-representative study found that $33.7 \%$ of the sample population received early ANC visits [3]. Regarding HIV tests during pregnancy, Ejigu et al. showed that in Ethiopia, more than 35\% of the sample population were tested for HIV [25], which is quite low compared with $75.4 \%$ in the present study. This is understandable based on the fact that Ethiopia (2.4\%) also has a far lower HIV rate compared with Mozambique $(13.2 \%$ as of 2015) [26]. In fact, However, the prevalence of Mozambique women taking HIV tests during pregnancy is lower compared with Uganda (81.5\%) [27] whose HIV rate is about half as much as in Mozambique [28].

In brief, the present study reports a low level of antenatal care use in Mozambique which requires special policy attention. Of particular concern is the prevalence of HIV testing, compared to the high incidence of HIV in the country the rate of HIV testing appears to be extremely low. Regarding the frequency and timing of ANC use, more than half of the women reported having at least 4 visits and making the first contact within the first trimester. Nonetheless, the number is still far from being universal as recommended by World Health Organisation. We found important sociodemographic inequalities for the use of these services, addressing whih can lead a better use of these life saving strategies. There are also several associations that deserve a special attention. For instance, women in the urban areas ANC were found to start ANC visit later than rural women, despite the fact that urban areas offer better access to healthcare. Although not deducible from the current findngs, this irregularity might be suugestive of urban poverty which is a growing issue across the continent. More research findings are necessary to understand this situation. Women who have outdoor employment were also less likely to get early ANC contact. In theory, working women are more empowered to access healthcare for themselves and for children. Therefore, this finding also remains to be interpreted from various perspectives such as healthcare benefits for pregnant women, granting leaves for checkup, and discrimination at healthcare centers. Collecting data on these factors are more complex and often challenging on a larger population, which is the likely reason why DHS studies do not collect data on these topics. New research models and techniques are required to meet these data limittaions.

Regarding the variables associated with the utilisation of the ANC services, several demographic (age), geographic (residency) and socioeconomic (education, wealth, ethnicity) factors were found be significantly associated with ANC service use. Women aged 20-24 years were found to have to lower odds of using timely and adequate ANC services compared with those in the lowest age group of 1519 years. The odds of early ANC visits were higher in the rural areas compared with urban, however, rural women had lower odds of using HIV tests during ANC visits. The causes behind the regional differences were not possible to measure from the current analysis due to lack of contextual data. In general, it is believed that the prevalence of healthcare use is more prevalent among urban population compared with their rural counterparts due to better health awareness, financial capacity, transportation facilities. The causes behind the urban-rural disparity in the use of ANC services in Mozambique should be given priority in future studies.

In line with previous studies, women with better education status and coming from higher wealth quintile households had higher odds of making early ANC visits and taking HIV test [24, 27]. Women's educational status is an important determinant of their social empowerment which itself is an important predictor of financial well-being and the capacity to access healthcare services. Education can promote healthcare seeking behaviour through its positive role on health awareness and improving self-efficacy [29]. Educated women are also more likely to be able to acquire information regarding the health risks associated with pregnancy which enable them to take preventive measures and reach out for professional helps such as counselling and medical tests. Similarly, being able to gather health information through modern electronic media such as radio, TV and internet can act as a strong enabling factor the healthcare use among women in the developing countries [30]. Strangely, the findings suggested no beneficial role of media access on making early and adequate ANC contact, although a positive association was found with taking HIV test. This supports the fact that women can gather knowledge on HIV transmission and risk factors and seek professional care during ANC as a means to prevent transmission 
of the virus to her child. Maternal health promotion programs in Mozambique should focus on developing strategies to improve the use of ANC services through leveraging mass media technology which is proving effective in many areas of public health interventions.

Women having employment in agriculture had a positive association with early ANC visits, but negative association with HIV test. Having an income source generally translates to better economic well-being and can act an enabling factor for utilisation of ANC services [27]. Maternal healthcare promotion programs should therefore pay special attention to non-working women who might remain deprived of the essential services owing to poor financial means. Another interesting finding was the ethnic disparity in the use of ANC services. Although the ethnicity is intrinsically a qualitative subject that requires qualitative investigation, our findings provide a glimpse of the relative inequality in the use of these services which can serve as the basis of further systematic investigation. Cultural factors influence health outcomes through various complex pathways, such as shaping people's concept of health and illness and the perceived need for seeking medical care they may not be familiar with [31]. Sociocultural inequality in accessing healthcare is an increasing concern in populations as ethnically diverse as in Mozambique [32]. Therefore, it is recommended that healthcare policy making process be made more sensitive to the cultural factors that are generally ignored in the primary care settings. Lastly, we found a significantly negative association between child wanted-ness and use of ANC services. This is perhaps because of the lack of awareness and anxiety associated with unplanned pregnancy that prevent women from taking active steps in taking care of her and the pregnancy issues. Given the high rate of HIV in Mozambique, routine HIV tests during pregnancy should be regarded as a special priority to prevent the spread of childhood HIV.

This study made use of a nationally-representative survey to report the prevalence of using ANC services in Mozambique. In the current literature, data on healthcare use women during pregnancy is rare and insignificant. From this aspect, the present findings make an important contribution to the knowledge base and provide the basis for further research in this area. Nonetheless, this study has several limitations to report. First of all, the data were cross-sectional and hence no causality can be inferred from the associations. The data were secondary and authors have no influence over the selection and measurement of the variables. As the data were self-reported, the chances of recall and reporting bias cannot be ignored [33, 34].

\section{Conclusion}

Findings of the present study highlights that although well above half of the women made at least four ANC visits and received HIV test during ANC, the proportions of women making early ANC contact was shockingly low (15\%). There are important inequalities in using the services across various factors, most notable of which are education, wealth status, ethnicity, access to media wanted-ness of the child. Women who fail to make early ANC visits are more likely to have potential risk conditions unchecked which may give rise to serious complications at the later stage, therefore, policy makers and implementers should make it a priority to increase awareness on the importance of ANC and ensure contacts with ANC through clinic visits are enforced in the first trimester. Addressing the inequalities among the sociodemographic groups should be given special attention in maternal health promotion programs.

\section{Abbreviations}

ANC: Antenatal care; DHS: Demographic Health Survey; IRB: Institutional Review Board; MDGS: Millennium Development Goals; USAID: United States Agency for International Development of the United America

\section{Acknowledgments \\ The authors thank the MEASURE DHS project for their support and for free access to the original data}

\section{Authors' contributions}

SY and GB contributed to the study design, the review of literature, manuscript conceptualisation and preparation. $\mathrm{OO}$ and KEO contributed to data analysis and critically reviewed the manuscript for its intellectual content. SY had final responsibility to submit for publication. All authors read and approved the final manuscript.

\section{Funding}

The authors have no support or funding to report.

\section{Availability of data and materials}

Data for this study were sourced from Mozambique Demographic and Health surveys (DHS) and available here: https://dhsprogram.com/data/ available-datasets.cfm

\section{Ethics approval and consent to participate}

For DHS data, ethical approval has obtained from the individual national institutions review board and by ICF International institutional review board. For children and adolescents, a parent or guardian must provide consent prior to participation. The description of consent process is available from: https://www.dhsprogram.com/What-We-Do/Protecting-the-Privacy-of-DHSSurvey-Respondents.cfm.

\section{Consent for publication}

Not applicable.

\section{Competing interests}

The authors declare that they have no competing interests.

\section{Author details}

'The George Institute for Global Health, 75 George Street, Oxford OX1 2BQ, United Kingdom. ${ }^{2}$ Social Aspects of Public Health, Human Sciences Research Council, Cape Town, South Africa. ${ }^{3}$ Department of Community Medicine, University of Jos, Jos, Nigeria. ${ }^{4}$ Department of Global Health, School of Public Health, University of Namibia, Namibia, South Africa. ${ }^{5}$ Department of Public Health, Faculty of Health Sciences, University of Fort Hare, Eastern Cape, South Africa. ${ }^{6}$ School of International Development and Global Studies, University of Ottawa, Ottawa, Ontario K1N 6N5, Canada. 
Received: 5 July 2019 Accepted: 27 September 2019

Published online: 15 October 2019

\section{References}

1. Black RE, Cousens S, Johnson HL, Lawn JE, Rudan I, Bassani DG, et al. Global, regional, and national causes of child mortality in 2008: a systematic analysis. Lancet Lond Engl. 2010;375:1969-87.

2. Cha S. The impact of the worldwide millennium development goals campaign on maternal and under-five child mortality reduction: 'where did the worldwide campaign work most effectively?'. Glob Health Action. 2017; 10. https://doi.org/10.1080/16549716.2017.1267961.

3. Yaya S, Bishwajit G, Ekholuenetale M, Shah V, Kadio B, Udenigwe O. Timing and adequate attendance of antenatal care visits among women in Ethiopia. PLoS One. 2017;12:e0184934.

4. The Public Health Importance of Antenatal Care. Facts Views Vis ObGyn. 2015;7:5-6.

5. Al-Ateeq MA, Al-Rusaiess AA. Health education during antenatal care: the need for more. Int J Women's Health. 2015;7:239-42.

6. Siddique AB, Perkins J, Mazumder T, Haider MR, Banik G, Tahsina T, et al. Antenatal care in rural Bangladesh: gaps in adequate coverage and content. PLoS One. 2018;13. https://doi.org/10.1371/journal.pone.0205149.

7. Benova L, Tunçalp Ö, Moran AC, Campbell OMR. Not just a number: examining coverage and content of antenatal care in low-income and middle-income countries. BMJ Glob Health. 2018;3. https://doi.org/10.1136/ bmjgh-2018-000779.

8. Mutabazi JC, Zarowsky C, Trottier H. The impact of programs for prevention of mother-to-child transmission of HIV on health care services and systems in sub-Saharan Africa - a review. Public Health Rev. 2017;38. https://doi.org/ 10.1186/s40985-017-0072-5.

9. Sama C-B, Feteh VF, Tindong M, Tanyi JT, Bihle NM, Angwafo FF. Prevalence of maternal HIV infection and knowledge on mother-to-child transmission of HIV and its prevention among antenatal care attendees in a rural area in Northwest Cameroon. PLoS One. 2017;12. https://doi.org/10.1371/journal. pone.0172102

10. Chi BH, Bolton-Moore C, Holmes CB. Prevention of mother-to-child HIV transmission within the continuum of maternal, newborn, and child health services. Curr Opin HIV AIDS. 2013:8:498-503.

11. Wudineh F, Damtew B. Mother-to-child transmission of HIV infection and its determinants among exposed infants on care and follow-up in Dire Dawa City. Eastern Ethiopia AIDS Res Treat. 2016;2016. https://doi.org/10.1155/ 2016/3262746

12. Anoje C, Aiyenigba B, Suzuki C, Badru T, Akpoigbe K, Odo M, et al. Reducing mother-to-child transmission of HIV: findings from an early infant diagnosis program in south-south region of Nigeria. BMC Public Health. 2012;12:184.

13. Saleem S, McClure EM, Goudar SS, Patel A, Esamai F, Garces A, et al. A prospective study of maternal, fetal and neonatal deaths in low- and middle-income countries. Bull World Health Organ. 2014;92:605-12.

14. Indonesia JC on RM and NM in, Development S, Affairs P and G, Council NR Sciences IA of. Maternal, Fetal, and Neonatal Mortality. National Academies Press (US); 2013. https://www.ncbi.nlm.nih.gov/books/NBK201704/. .

15. Sankar MJ, Natarajan CK, Das RR, Agarwal R, Chandrasekaran A, Paul VK. When do newborns die? A systematic review of timing of overall and cause-specific neonatal deaths in developing countries. J Perinatol. 2016; 36(Suppl 1):S1-11.

16. Adjiwanou V, LeGrand T. Gender inequality and the use of maternal healthcare services in rural sub-Saharan Africa. Health Place. 2014;29:67-78.

17. Fekadu GA, Kassa GM, Berhe AK, Muche AA, Katiso NA. The effect of antenatal care on use of institutional delivery service and postnatal care in Ethiopia: a systematic review and meta-analysis. BMC Health Serv Res. 2018; 18. https://doi.org/10.1186/s12913-018-3370-9.

18. Gouda HN, Hodge A, Bermejo R III, Zeck W. Jimenez-Soto E. The Impact of Healthcare Insurance on the Utilisation of Facility-Based Delivery for Childbirth in the Philippines. https://journals.plos.org/plosone/article?id= 10.1371/journal.pone.0167268.

19. Sialubanje C, Massar K, Hamer DH, Ruiter RAC. Personal and environmental predictors of the intention to use maternal healthcare services in Kalomo. Zambia Health Educ Res. 2014;29:1028-40.

20. Ghose B, Feng D, Tang S, Yaya S, He Z, Udenigwe O, et al. Women's decision-making autonomy and utilisation of maternal healthcare services: results from the Bangladesh demographic and health survey. BMJ Open. 2017;7:e017142.
21. Tesfaye G, Chojenta C, Smith R, Loxton D. Application of the AndersenNewman model of health care utilization to understand antenatal care use in Kersa District. Eastern Ethiopia PLOS ONE. 2018;13:e0208729.

22. Sakshaug JW, West BT. Important considerations when analyzing health survey data collected using a complex sample design. Am J Public Health. 2014;104:15-6.

23. Dickson KS, Darteh EKM, Kumi-Kyereme A, Ahinkorah BO. Determinants of choice of skilled antenatal care service providers in Ghana: analysis of demographic and health survey. Matern Health Neonatol Perinatol. 2018;4. https://doi.org/10.1186/s40748-018-0082-4.

24. Saad-Haddad G, DeJong J, Terreri N, Restrepo-Méndez MC, Perin J, Vaz L, et al. Patterns and determinants of antenatal care utilization: analysis of national survey data in seven countdown countries. J Glob Health. 6. doi: https://doi.org/10.7189/jogh.06.010404.

25. Ejigu $Y$, Tadesse B. HIV testing during pregnancy for prevention of motherto-child transmission of HIV in Ethiopia. PLoS One. 2018;13. https://doi.org/ 10.1371/journal.pone.0201886.

26. Frey A. HIV prevalence in Mozambique rises about two percent. Mozambique. https://clubofmozambique.com/news/hiv-prevalence-inmozambique-rises-about-two-percent/.

27. Gunn JKL, Asaolu IO, Center KE, Gibson SJ, Wightman P, Ezeanolue EE, et al. Antenatal care and uptake of HIV testing among pregnant women in subSaharan Africa: a cross-sectional study. J Int AIDS Soc. 2016;19. https://doi. org/10.7448/IAS.19.1.20605.

28. HIV and AIDS in Uganda. AVERT. 2015. https:/www.avert.org/professionals/ hiv-around-world/sub-saharan-africa/uganda. Accessed 22 May 2019.

29. Yaya S, Bishwajit G, Ekholuenetale M, Shah V. Awareness and utilization of community clinic services among women in rural areas in Bangladesh: a cross-sectional study. PLoS One. 2017;12:e0187303.

30. Tsawe M, Moto A, Netshivhera T, Ralesego L, Nyathi C, Susuman AS. Factors influencing the use of maternal healthcare services and childhood immunization in Swaziland. Int J Equity Health. 2015;14. https://doi.org/10. 1186/s12939-015-0162-2.

31. Coast E, Jones E, Portela A, Lattof SR. Maternity care services and culture: a systematic global mapping of interventions. PLoS One. 2014;9. https://doi. org/10.1371/journal.pone.0108130.

32. Audet CM, Burlison J, Moon TD, Sidat M, Vergara AE, Vermund SH. Sociocultural and epidemiological aspects of HIV/AIDS in Mozambique. BMC Int Health Hum Rights. 2010;10:15.

33. Yaya S, Uthman OA, Okonofua F, Bishwajit G. Decomposing the rural-urban gap in the factors of under-five mortality in sub-Saharan Africa? Evidence from 35 countries. BMC Public Health. 2019;19(1):616.

34. Yaya S, Gunawardena N, Bishwajit G. Association between intimate partner violence and utilization of facility delivery services in Nigeria: a propensity score matching analysis. BMC Public Health. 2019 Aug 17;19(1):1131. https:// doi.org/10.1186/s12889-019-7470-1.

\section{Publisher's Note}

Springer Nature remains neutral with regard to jurisdictional claims in published maps and institutional affiliations.

Ready to submit your research? Choose BMC and benefit from:

- fast, convenient online submission

- thorough peer review by experienced researchers in your field

- rapid publication on acceptance

- support for research data, including large and complex data types

- gold Open Access which fosters wider collaboration and increased citations

- maximum visibility for your research: over $100 \mathrm{M}$ website views per year

At BMC, research is always in progress.

Learn more biomedcentral.com/submissions 